\title{
Comprehensive Analysis of Effect of Submergence on Rice Grain Quality
}

\author{
Mubashar Hussain ${ }^{1 *}$, Nauman Liaqat ${ }^{2}$, Muhammad Bilal ${ }^{3}$ and Hamza Armghan Noushahi ${ }^{4}$ \\ ${ }^{1}$ Rice Research Institute, Kala Shah Kaku, Lahore, Pakistan \\ ${ }^{2}$ Department of Agronomy, Pakistan \\ ${ }^{3}$ Department of Entomology, Pakistan \\ ${ }^{4}$ College of Plant Science and Technology, China \\ *Corresponding author: Mubashar Hussain, Rice Research Institute, Kala Shah Kaku, Lahore, Pakistan
}

\begin{abstract}
Flash flooding is one of the major problems in rice growing areas of South and Southeast Asia and it severely limits rice yield in these areas. This experiment was performed to check cultivated varieties with resistance against submergence and check best varieties which give better grain quality after affected by submergence. Chenab Basmati one of the cultivated varieties proved best performer in this experiment and is recommended for cultivation in areas affected by flood in Pakistan.
\end{abstract}

\section{Introduction}

Rice is one of the important crops in cereals. Rice is staple food of $50 \%$ population all around the world. Its higher production and good taste are two main parameters for many breeding projects but, in contrary to disease and insect resistance, grain yield and quality are both managed by quantitative trait loci (QTLs) highlighting continuous phenotypic difference in rice offspring [1]. So, it is hard for breeders to upgrade rice grain yield and quality using conventional techniques, due to a lack of distinct phenotypic segregation in the offspring. As rice grain quality is an endosperm characteristic, its heredity can be more complex because the genetic expression of an endosperm trait in cereal seeds is conditioned not only by the triploid endosperm genotype, but also by the diploid maternal genotype and any additional possible cytoplasmic differences [2-4]. Quality of rice grain comprises of cooking, milling, appearance and nutritional qualities. But appearance and cooking quality is mostly focused by people [5]. The appearance quality is often judged in China by the percentage of grain with a white core and a square of white core. Climate change is enhancing submergence stress, which is one of the main hurdles in increasing rice yield in rice growing areas of world. The most common and devastating type of flooding is short-time inundation (up to 2 weeks), also known as flash floods. 20 million hectare of rice growing area is affected by this kind of flooding in Asia (excepted China) as well as many lowland areas of Africa [6-10]. Moreover, due to disastrous effects from climate change, these seasonal flash floods are adversely unpredictable and can occur at any growth period of the rice crop [11]. Rice is submerged in monsoon season in South and Southeast Asia, which adversely limits rice yield and causes one billion U.S dollars losses annually [12].

\section{Material and Methods}

There were two sets of genotypes used in this experiment $1^{\text {st }}$ set was grown in control environment, while $2^{\text {nd }}$ was grown in submerged environment in water tank (Table 1). Screw guage was used to measure the grain dimensions like length, width and breadth of controlled and submerged material to study the effect of submergence on grain length, width, breadth and other quality parameters (Table 2). 
Table 1: Quality parameters data for submerged material ( $\mathrm{mm})$.

\begin{tabular}{|c|c|c|c|c|c|c|c|c|c|}
\hline \multirow{2}{*}{ Entries } & \multirow{2}{*}{$\begin{array}{c}\text { R1 } \\
\text { Length }\end{array}$} & \multirow[b]{2}{*}{ Width } & \multirow[b]{2}{*}{ Breadth } & \multicolumn{3}{|c|}{$\mathbf{R 2}$} & \multicolumn{3}{|c|}{ R3 } \\
\hline & & & & Length & Width & Breadth & Length & Width & Breadth \\
\hline V1 & 5.4 & 1 & 1.5 & 5.3 & 1.7 & 2 & 6.5 & 1.6 & 2.4 \\
\hline $\mathrm{V} 2$ & 6.3 & 1.5 & 2 & 6.3 & 1.5 & 1.8 & 7.2 & 1.7 & 2.2 \\
\hline V4 & 5.7 & 1.3 & 2.2 & 5.6 & 1.2 & 1.7 & 5.7 & 1.4 & 2.2 \\
\hline V5 & 6.8 & 1.6 & 2.2 & 6.7 & 1.6 & 2 & 7.1 & 2.1 & 2.5 \\
\hline V6 & 7.2 & 1.6 & 2.2 & 7 & 1.6 & 2.3 & 6.7 & 1.7 & 2.2 \\
\hline V7 & 71 & 1.6 & 2.5 & 7.2 & 1.7 & 2.6 & 5.5 & 1.5 & 2.6 \\
\hline V8 & 5 & 1.4 & 1.7 & 5.6 & 1.3 & 2.2 & 5.4 & 1.7 & 1.8 \\
\hline V9 & 4.6 & 1.2 & 1.4 & 6 & 1.3 & 1.8 & 5.2 & 1.4 & 1.8 \\
\hline V10 & 6.4 & 1.5 & 1.7 & 5.4 & 1.3 & 1.8 & 5.7 & 1.5 & 1.8 \\
\hline $\mathrm{V} 11$ & 5.9 & 1.2 & 1.8 & 5.7 & 1.3 & 1.7 & 5.2 & 1.7 & 2.2 \\
\hline V12 & 6 & 1.4 & 1.9 & 6.2 & 1.5 & 1.7 & 6.4 & 1.3 & 1.6 \\
\hline V13 & 6.6 & 1.7 & 2.2 & 5.7 & 1.7 & 2.3 & 6 & 1.5 & 2.3 \\
\hline V14 & 6.8 & 1.6 & 2.2 & 7.3 & 1.8 & 1.8 & 7 & 1.6 & 2.2 \\
\hline V15 & 6.1 & 1.6 & 2.2 & 5.7 & 1.7 & 2.2 & 6.1 & 1.3 & 1.8 \\
\hline V16 & 7.6 & 2.3 & 2.6 & 6.6 & 2 & 2.7 & 7.3 & 2.3 & 2.4 \\
\hline V17 & 7.4 & 2.3 & 2.7 & 7.5 & 2.2 & 2.6 & 7.4 & 2.3 & 2.4 \\
\hline V18 & 7.1 & 2.1 & 2.3 & 7.3 & 1.8 & 2.5 & 7.5 & 2.3 & 2.6 \\
\hline V19 & 6.8 & 1.4 & 2.3 & 6.7 & 1.7 & 2.2 & 6.2 & 1.8 & 2.3 \\
\hline V20 & 6.7 & 2 & 2.3 & 7.3 & 2.2 & 2.7 & 7.2 & 2.4 & 2.4 \\
\hline V21 & 6.8 & 2.2 & 2.4 & 7 & 1.8 & 2.5 & 6.9 & 2 & 2.4 \\
\hline V22 & 7.2 & 1.7 & 2.6 & 7.3 & 1.7 & 2.2 & 6.8 & 1.6 & 2.3 \\
\hline V23 & 6.9 & 2 & 2.4 & 6.9 & 1.4 & 2 & 7 & 1.6 & 2.2 \\
\hline V24 & 6.7 & 1.5 & 2 & 7.2 & 1.6 & 2.3 & 6.4 & 1.7 & 2 \\
\hline V25 & 5.8 & 1.7 & 2.3 & 6 & 1.6 & 1.8 & 5.6 & 1.9 & 2.7 \\
\hline V26 & 6.7 & 1.5 & 2.2 & 6.3 & 1.7 & 2.4 & 6.6 & 1.9 & 2.3 \\
\hline V27 & 6.4 & 1.7 & 2.4 & 6.7 & 1.5 & 2.3 & 6.7 & 1.8 & 2.2 \\
\hline V28 & 7.5 & 1.7 & 1.8 & 6.3 & 1.5 & 1.7 & 6.2 & 1.7 & 2.2 \\
\hline V29 & 6.7 & 1.2 & 1.8 & 6.6 & 1.2 & 1.6 & 7 & 1.5 & 2 \\
\hline V30 & 6.7 & 1.7 & 2.3 & 6.3 & 1.8 & 2.4 & 6.7 & 1.6 & 2.5 \\
\hline V31 & 7.2 & 1.7 & 2.5 & 7.2 & 1.8 & 2.5 & 7.2 & 1.8 & 2.4 \\
\hline V32 & 7.4 & 2.3 & 2.6 & 7.2 & 1.8 & 2.4 & 7.2 & 1.5 & 1.8 \\
\hline V33 & 6.8 & 1.8 & 2.4 & 6.7 & 1.3 & 2.3 & 6.3 & 1.2 & 2 \\
\hline V34 & 6.3 & 1.4 & 2.4 & 6 & 1.7 & 2.2 & 5.2 & 1.3 & 1.6 \\
\hline V35 & 6.7 & 2 & 2.2 & 6.7 & 1.7 & 2 & 6.5 & 1.7 & 2.2 \\
\hline V36 & 6.2 & 1.6 & 2.3 & 7 & 2 & 2.6 & 7.1 & 2.2 & 2.6 \\
\hline V37 & 6.8 & 2.3 & 3 & 7.5 & 2.2 & 2.6 & 7.2 & 2.1 & 3.2 \\
\hline V38 & 8 & 2.7 & 3 & 7.7 & 2.6 & 3.5 & 7.1 & 2.3 & 2.7 \\
\hline V39 & 6.8 & 1.8 & 2.4 & 5.7 & 1.2 & 2.3 & 5.3 & 0.8 & 1.4 \\
\hline V40 & 7.6 & 1.8 & 2.5 & 7.2 & 1.6 & 2.3 & 7.1 & 1.5 & 2.3 \\
\hline V41 & 7.1 & 1.7 & 2 & 6.6 & 1.5 & 2.3 & 6.7 & 1.3 & 1.8 \\
\hline V42 & 6.8 & 1.6 & 2.2 & 6.7 & 1.6 & 2 & 7 & 2.1 & 2.5 \\
\hline V43 & 6.8 & 1.8 & 2.5 & 7.2 & 1.7 & 2.4 & 7 & 1.7 & 2.4 \\
\hline V44 & 6 & 1.6 & 2.3 & 6.1 & 1.4 & 2.2 & 6 & 1.2 & 2.2 \\
\hline V45 & 6.5 & 1.7 & 2.3 & 7 & 1.5 & 2.4 & 6.3 & 1.6 & 2 \\
\hline V46 & 7.7 & 2 & 2.4 & 6.6 & 1.8 & 2.7 & 6.2 & 1.7 & 2.6 \\
\hline
\end{tabular}




\begin{tabular}{|c|c|c|c|c|c|c|c|c|c|}
\hline V47 & 7.4 & 2 & 2.6 & 6.6 & 1.4 & 2.4 & 7.3 & 1.8 & 2.5 \\
\hline V48 & 5.7 & 1.3 & 2.2 & 7.3 & 1.4 & 2 & 5.4 & 1.5 & 1.7 \\
\hline V49 & 6.7 & 1.4 & 2 & 6.8 & 1.4 & 2 & 7.1 & 1.6 & 1.8 \\
\hline V50 & 7.8 & 1.8 & 2.7 & 7.5 & 2.1 & 2.8 & 7.2 & 2.3 & 2.6 \\
\hline
\end{tabular}

Table 2: Quality parameters data of control material (mm).

\begin{tabular}{|c|c|c|c|c|c|c|c|c|c|}
\hline \multirow[t]{2}{*}{ Entries } & \multicolumn{3}{|c|}{ R1 } & \multicolumn{3}{|c|}{$\mathbf{R 2}$} & \multicolumn{3}{|c|}{ R3 } \\
\hline & Length & Width & Breadth & Length & Width & Breadth & Length & Width & Breadth \\
\hline V1 & 7.4 & 2.3 & 2.5 & 7.5 & 1.7 & 2.2 & 7.4 & 2 & 2.5 \\
\hline V2 & 8.4 & 2.5 & 3.3 & 8.2 & 2.6 & 2.7 & 8.3 & 2.7 & 3.1 \\
\hline V3 & 6.3 & 2.2 & 2.7 & 6.6 & 2.4 & 2.6 & 6.9 & 2.5 & 3.2 \\
\hline V4 & 7.5 & 3.1 & 3.4 & 7.3 & 3 & 3.1 & 6.8 & 2.5 & 2.5 \\
\hline V5 & 6.2 & 1.3 & 2.2 & 5.8 & 1.4 & 2.1 & 6.5 & 1.8 & 2.3 \\
\hline V6 & 7.5 & 1.8 & 2.4 & 7.3 & 2.1 & 2.2 & 7.5 & 1.9 & 2.2 \\
\hline V7 & 7.8 & 2.9 & 3.1 & 8.2 & 2.8 & 3.4 & 8.3 & 2.7 & 3.7 \\
\hline V8 & 7.6 & 2.4 & 3.1 & 7.7 & 2.5 & 3.3 & 7.9 & 2.4 & 3.1 \\
\hline V9 & 6.4 & 2.4 & 3 & 6.7 & 2.7 & 3.2 & 6.5 & 2.7 & 3.1 \\
\hline V10 & 6.4 & 2.5 & 3.2 & 7.3 & 2.7 & 3.5 & 7 & 2.6 & 3.5 \\
\hline V11 & 6.2 & 1.8 & 2.5 & 6.4 & 2.1 & 2.6 & 6.2 & 2 & 2.6 \\
\hline V12 & 7.7 & 2.7 & 3.1 & 7.5 & 2.4 & 3.1 & 7.9 & 2.5 & 3.1 \\
\hline V14 & 6.7 & 1.8 & 2.4 & 7.2 & 1.9 & 2.5 & 8 & 1.9 & 2.3 \\
\hline V16 & 6.6 & 1.7 & 2.3 & 6.7 & 1.7 & 2.2 & 7.2 & 1.9 & 2.2 \\
\hline V19 & 8.2 & 2.9 & 3.5 & 7.8 & 2.7 & 2.8 & 8.5 & 2.6 & 3.2 \\
\hline V20 & 7.1 & 1.6 & 2.2 & 7.2 & 1.4 & 2.3 & 7.1 & 1.7 & 2.5 \\
\hline V21 & 7.4 & 1.8 & 2.4 & 7.2 & 1.3 & 2.4 & 7 & 1.8 & 2.3 \\
\hline V24 & 6.3 & 1.9 & 2.2 & 6.3 & 1.6 & 2.1 & 6.3 & 1.8 & 2.1 \\
\hline V25 & 6.3 & 1.6 & 2.5 & 6.4 & 1.7 & 2.1 & 6.4 & 1.2 & 2.2 \\
\hline V26 & 7.3 & 1.9 & 2.4 & 7.1 & 1.8 & 2.1 & 7.2 & 1.6 & 2.4 \\
\hline V27 & 7.1 & 1.7 & 2.4 & 6.6 & 1.7 & 2.2 & 7.1 & 2.1 & 2.5 \\
\hline V28 & 7.8 & 1.9 & 2.1 & 7.8 & 1.7 & 2.3 & 6.9 & 1.4 & 2.3 \\
\hline V30 & 7.4 & 1.8 & 2.5 & 7.3 & 1.6 & 2.4 & 6.8 & 1.7 & 2.2 \\
\hline V31 & 7.7 & 2.1 & 2.4 & 7.2 & 1.9 & 2.4 & 7.9 & 1.8 & 2.4 \\
\hline V32 & 7.2 & 1.7 & 2.6 & 7.2 & 1.4 & 2.5 & 7.5 & 1.9 & 2.3 \\
\hline V33 & 8.3 & 3.2 & 3.6 & 8.4 & 3 & 3.6 & 8.1 & 3.2 & 3.5 \\
\hline V35 & 7.4 & 1.7 & 2.2 & 7.3 & 1.5 & 2.2 & 7 & 1.8 & 2.3 \\
\hline V37 & 7.4 & 1.7 & 2.3 & 7.7 & 1.6 & 2.1 & 7.6 & 1.7 & 2.2 \\
\hline V38 & 7.7 & 1.7 & 2 & 7.3 & 1.7 & 2.2 & 6.5 & 1.6 & 2.2 \\
\hline V40 & 7.5 & 1.6 & 2.2 & 7.2 & 1.4 & 2.2 & 7.6 & 1.6 & 2.1 \\
\hline V42 & 7.1 & 1.6 & 2.3 & 6.5 & 1.6 & 2.4 & 7 & 1.5 & 2.2 \\
\hline V43 & 6.8 & 1.6 & 2.1 & 7.3 & 1.9 & 2.4 & 7.1 & 1.9 & 2.3 \\
\hline V44 & 6.3 & 1.6 & 2.4 & 6.6 & 1.7 & 2.5 & 5.9 & 1.6 & 2.1 \\
\hline V45 & 7.2 & 1.6 & 2.3 & 7.1 & 1.6 & 2.2 & 6.5 & 1.5 & 2.1 \\
\hline V46 & 7.4 & 1.6 & 2.4 & 7 & 1.8 & 2.4 & 7.1 & 1.7 & 2.2 \\
\hline V47 & 7.1 & 2.8 & 3.5 & 7.2 & 3.1 & 3.7 & 6.5 & 3 & 3.4 \\
\hline V49 & 6.8 & 1.5 & 2.2 & 7.4 & 1.6 & 2.1 & 6.7 & 1.8 & 2.3 \\
\hline V50 & 5.8 & 1.4 & 1.7 & 6.5 & 1.7 & 2.5 & 6.2 & 1.7 & 2.1 \\
\hline
\end{tabular}




\section{Material}

(Table 3).

\section{Table 3.}

\begin{tabular}{|c|c|}
\hline Code NO & Variety Name \\
\hline V1 & IR-6 \\
\hline V2 & Super Basmati \\
\hline V3 & NIAB IRRI 9 \\
\hline V4 & KSK-133 \\
\hline V5 & KSK-434 \\
\hline V6 & Basmati 370 \\
\hline V7 & Basmati Pak \\
\hline V8 & Basmati 198 \\
\hline V9 & Basmati 385 \\
\hline V10 & KS-282 \\
\hline V11 & Basmati 2000 \\
\hline V12 & Shaheen Basmati \\
\hline V13 & Basmati 515 \\
\hline V14 & PS-2 \\
\hline V15 & PK 386 \\
\hline V16 & Kisan Basmati \\
\hline V17 & Shadab \\
\hline V18 & Punjab Basmati \\
\hline V19 & PK 8892-4-2-1-1 \\
\hline V20 & PK 8892-4-1-3-1 \\
\hline V21 & PK 9194 \\
\hline V22 & RRI 3 \\
\hline V23 & PK BB15-1 \\
\hline V24 & PK BB15-6 \\
\hline V25 & PKBB8 \\
\hline V26 & PK 10355 \\
\hline V27 & Kashmir Basmati \\
\hline V28 & DR-82 \\
\hline V29 & DR-83 \\
\hline V30 & Sada Hayat \\
\hline V31 & DR-92 \\
\hline V32 & Khushbo-95 \\
\hline V33 & Chenab Basmati \\
\hline V34 & Shua-92 \\
\hline V35 & Sarshar \\
\hline V36 & Jhona 349 \\
\hline V37 & Mushkan-41 \\
\hline V38 & Sathra-278 \\
\hline V39 & Mahlar-346 \\
\hline V40 & Palman 246 \\
\hline V41 & C-622 \\
\hline V42 & IRRI Pak (IR8) \\
\hline V43 & PK177 \\
\hline V44 & KS282 \\
\hline
\end{tabular}




\begin{tabular}{|c|c|}
\hline V45 & Kashmir Nafees \\
\hline V46 & Rachna Basmati \\
\hline V47 & Jhona MF \\
\hline V48 Pakhal & Swat 1 \\
\hline V49 & Swat 2 \\
\hline V50 & \\
\hline
\end{tabular}

\section{Results and Discussion}

Submerged genotypes showed great amount of difference among each other in three characteristics of grain width, length and breadth. Genotype 2 showed highest value of mean in case of grain length and maintained $8^{\text {th }}$ rank in grain width. On other hand genotype 5 showed less gain in grain length. In case of grain width genotype 33 gave maximum width and ranked $2^{\text {nd }}$ in case of grain length. Similarly, smallest mean of grain width was shown by genotype 42 . Genotype 33 highlighted maximum grain breadth while genotype 31 was lowest ranked with minimum grain breadth.

Overall, genotype 33 gave best results and proved best performer under submergence because it secured $1^{\text {st }}$ position in case of grain width and breadth and at $2^{\text {nd }}$ position in case of grain length. On other hand, if we compare the grain width, breadth and length of genotypes 33 between controls and submerged, we conclude that submerged genotype is enough close to control. So, genotype 33 (Chenab Basmati) is recommended for cultivation in areas affected by flash flooding in Pakistan. While genotype 42 was poor performer because of minimum values for grain length, width and breadth in comparison to all submerged genotypes as well as its control.

\section{Appendix}

\section{(Appendix 1)}

\section{References}

1. Akinwale MG, Akinyele BO, Odiyi AC, Nwilene F, Gregorio G, et al. (2012) Phenotypic screening of Nigerian rainfed lowland mega rice varieties for submergence tolerance. In Proceedings of the world congress on Engineering 1: 4-6.

2. Fukao T, Yeung E, Bailey Serres J (2011) The submergence tolerance regulator SUB1A mediates crosstalk between submergence and drought tolerance in rice. The Plant Cell pp. 110.

3. Neeraja CN, Maghirang Rodriguez R, Pamplona A, Heuer S, Collard BC, et al. (2007) A marker-assisted backcross approach for developing submergence-tolerant rice cultivars. Theoretical and Applied Genetics 115(6): 767-776.

4. Manangkil OE, Vu HTT, Yoshida S, Mori N, Nakamura C (2008) A simple, rapid and reliable bioassay for evaluating seedling vigor under submergence in indica and japonica rice (Oryza sativa L.). euphytica 163(2): 267-274.

5. Manzanilla DO, Paris TR, Vergara GV, Ismail AM, Pandey S, et al. (2011) Submergence risks and farmers preferences: implications for breeding Sub1 rice in Southeast Asia. Agricultural Systems 104(4): 335-347.

6. Nawarathna RN, Perera ALT, Samarasinghe WLG (2014) Screening of BC1F1 population (BG 379-2/IR 07F102//BG 379-2) of rice (Oryza sativa $L$.) for submergence tolerance using molecular markers. Journal of Agricultural Sciences 9(3).

7. Ranawake AL, Amarasinghe UGS, Senanayake SGJN (2014) Submergence tolerance of some modern rice cultivars at seedling and vegetative stages. Journal of Crop and Weed 10(2): 240-247.

8. Shelley IJ, Takahashi Nosaka M, Kano Nakata M, Haque MS, Inukai Y (2016) Rice cultivation in Bangladesh: present scenario, problems, and prospects. Journal of International Cooperation for Agricultural Development 14: 20-29.

9. Singh S, Mackill DJ, Ismail AM (2014) Physiological basis of tolerance to complete submergence in rice involves genetic factors in addition to the SUB1 gene. AoB Plants 3: 6 .

10. Nelson Erik (2009) Modeling multiple ecosystem services, biodiversity conservation, commodity production, and tradeoffs at landscape scales. Frontiers in Ecology and the Environment 7(1): 4-11.

11. Tews J, Brose U, Grimm V, Tielbörger K, Wichmann MC, et al. (2004) Animal species diversity driven by habitat heterogeneity/diversity: the importance of keystone structures. Journal of biogeography 31(1): 7992.

12. Das KK, Panda D, Sarkar RK, Reddy JN, Ismail AM (2009) Submergence tolerance in relation to variable floodwater conditions in rice. Environmental and Experimental Botany 66(3): 425-434. 
(c) 9

This work is licensed under Creative Commons Attribution 4.0 License

To Submit Your Article Click Here: Submit Article

DOI: $10.32474 /$ CIACR.2018.05.000223

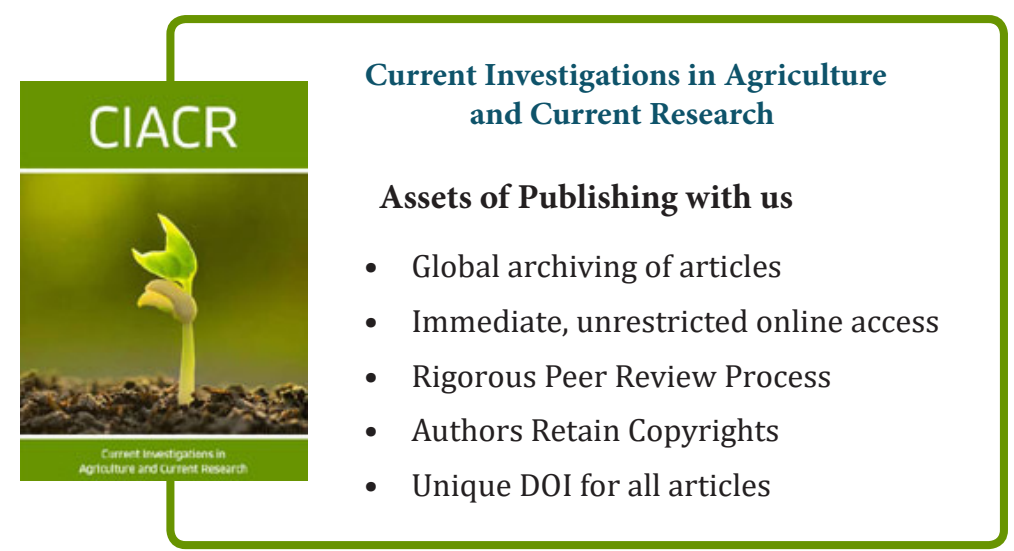

